\title{
A Deep Machine Learning Method for Concurrent and Interleaved Human Activity Recognition
}

\author{
Keshav Thapa ${ }^{\circledR}$, Zubaer Md. Abdullah Al, Barsha Lamichhane and Sung-Hyun Yang * \\ Department of Electronics Engineering, Kwangwoon University, Seoul 139-701, Korea; \\ kshavthapa@kw.ac.kr (K.T.); abdullah@kw.ac.kr (Z.M.A.A.); barshaer@kw.ac.kr (B.L.) \\ * Correspondence: shyang@kw.ac.kr
}

Received: 17 September 2020; Accepted: 6 October 2020; Published: 12 October 2020

\begin{abstract}
Human activity recognition has become an important research topic within the field of pervasive computing, ambient assistive living (AAL), robotics, health-care monitoring, and many more. Techniques for recognizing simple and single activities are typical for now, but recognizing complex activities such as concurrent and interleaving activity is still a major challenging issue. In this paper, we propose a two-phase hybrid deep machine learning approach using bi-directional Long-Short Term Memory (BiLSTM) and Skip-Chain Conditional random field (SCCRF) to recognize the complex activity. BiLSTM is a sequential generative deep learning inherited from Recurrent Neural Network (RNN). SCCRFs is a distinctive feature of conditional random field (CRF) that can represent long term dependencies. In the first phase of the proposed approach, we recognized the concurrent activities using the BiLSTM technique, and in the second phase, SCCRF identifies the interleaved activity. Accuracy of the proposed framework against the counterpart state-of-art methods using the publicly available datasets in a smart home environment is analyzed. Our experiment's result surpasses the previously proposed approaches with an average accuracy of more than $93 \%$.
\end{abstract}

Keywords: concurrent; interleaved; RNN; BiLSTM; SCCRF; activity recognition; Smart Home

\section{Introduction}

Human activity recognition has been a foremost research field since last decades due to advancements in technologies and its applicability in different domains like home automation, elderly care [1], telemedicine services [2], ubiquitous computing [3], and so forth. Among them, Smart homes embedded with many most uncomplicated, portable, fastest sensors have gained much attention in the field of ambient assisted living for prospering the quality of resident's life [4,5]. Based on the sensors used, wearable sensors and external sensors are used to identify the activity. The wearable sensor attached to the human body measure the signal patterns obtained through a movement of the human body. External sensors installed into different locations of the environment helps to recognized activity according to its generated value. One importance of activity recognition is to provide information on a human's behavior that allows computing systems to assist them in their daily tasks proactively. However, recognizing activities is a challenging task because human activities are complex and uncertain. Thus, only a few approaches address complex activity recognition [6]. Many of these approaches detect single user-single activity at a time, and a consecutive sequence of sensors data can achieve that. Nevertheless, multiple-user multiple-activity exists within a single sequence of actions as a concurrent and interleaving activity [7]. The recognizing of two activities concurrently through an observed activity sequence is called concurrent activity. Likewise, if one activity is paused (store for a short time) and then resumes after executing another activity is called interleaved activity. Recognition of activity mainly consists of three steps, as shown in Figure 1. First, the sliding window technique is applied to segments a stream of raw sensor data. More of all window shifts a fixed 
time-length or a set number of sensor data along the stream with overlapping or non-overlapping between adjacent segments. In the second step, we extract features (e.g., max, min, mean, variance, and entropy) from each segment and represent the feature vector's raw signals. The last step is called training and testing [8-10]. Recognition of activities needed detailed user specification, a large amount of training data, sophisticated network, and time-varying sequential data processing. The machine learning method solves these kinds of problems.

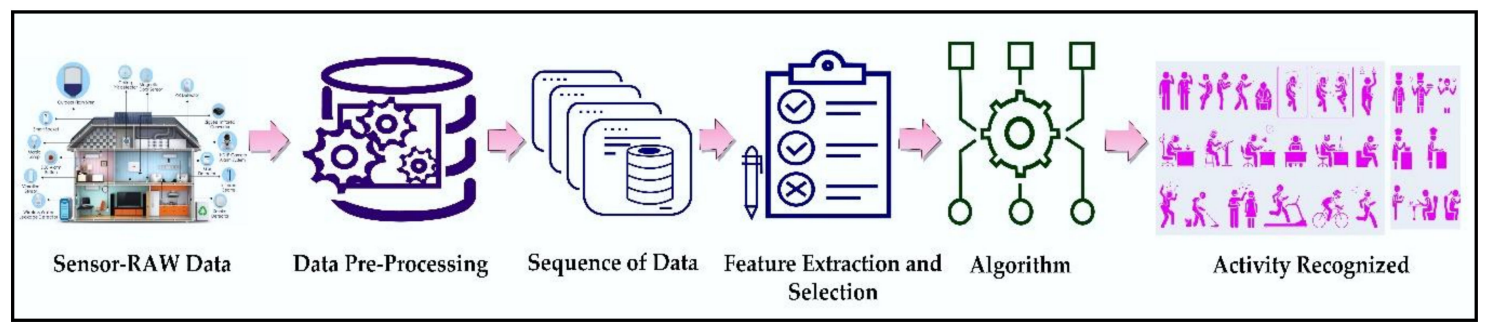

Figure 1. General Process for Activity Recognition.

Necessarily, many different probabilistic and non-probabilistic machine learning methods [11-13] have been hot-figure for activity recognition in recent years. However, difficulties confronted by traditional machine learning approaches are overcome by deep learning and led to numerous enhancement in activity recognition. A deep learning method called LSTM, A recurrent neural network, is used to recognize published by [14]. These techniques have already better performed than other machine learning algorithms in many applications like speech recognition [15], text recognition [16], image recognition [17] computer vision, including human activity recognition. The skip chain CRF [18] established the correlation between non-consecutive identical activities in the environment. This skip-chain is a probabilistic model; it has an advantage in recognizing activity naturally and conveniently. Therefore this unique feature of learning sequential, feedback connections, and storage ability of LSTM and recognizing identical features or data along long edges of SCCRF makes them recognize the composite activities. In this paper, we introduce the approach that contains a single platform by combining the features of BiLSTM and SCCRF to recognize these complex activities. This proposed approach could have been the first proposed so far to date. Our proposed method highlights;

- We present the two-phase recognition method. The first phase is capable of automatically learning concurrent feature representations and modeling the temporal dependencies between their activation to detect the concurrent activity with a deep learning framework composed of Bi-directional LSTM. The second phase explicitly models dependencies between distant activity and turns out to be particularly useful in interleaved activity detection using SCCRF.

- A feature-learning structure can directly learn spatial-temporal features from the raw data via LSTM structures, which requires neither manual feature selection nor classifier selection.

- The proposed framework can be applied seamlessly to different recognition modalities and other recognition platforms.

- The system adopts the raw sensor data with less preprocessing, which makes it exceptionally comfortable and general.

- We compare the performance of our framework to publically available datasets from Kasteren and Kyoto (WSU).

- The results depicted by our proposed framework outperforms published results on recognition of concurrent and interleaved activity.

- The proposed approach can classify the variable window ranges of human activities. Utilizing the LSTM to read variable window ranges, sequences of input sensors data can later recognize the entire window segment's activity. 
The remainder of the paper is organized as follows; related work is in Section 2. Section 3 illustrates the materials and method related to the proposed approach. Part 4 is about our experimental configuration for the activity recognition method. The activity recognition performance analysis is described in Section 5. Section 6 describes a conclusion.

\section{Related Work}

Activity recognition aims to recognize a user's high-level activity from different sensors or actions the user performed. In this case, they mostly like to have either logical-based approaches or probabilistic approaches. [19] Explained the logic-based approach, which provides a formal theory of activity recognition. Still, these approaches have limited inconsistent activity, unable to handle uncertainty, single activity recognition, maximum noise appearance, and significant rule of conduct. A procedure tries to solve the multiple-activity recognition problem in [20]. In their approach, a finite state machine creates transitions between different activity states, i.e., interleaving activity. However, this also fails to handle uncertainty. Concurrent activity recognition using a single classifier, encoding a binary output vector, has gained little attention, but its timing problem overruled activity's concurrency.

In the millennium, following the success of machine learning [21] and deep learning [22], wearable sensor-based activity recognition came in noticed. Restricted Boltzmann machine RBM on deep learning methods has not significantly improved deep belief network (DBNs) [23] with multiple layers. The Hidden Markov models also have been exploited above RBM layers on DBN based models harness temporal sequences in human activities. However, HMM performs well on limited numbers of hidden states and trying to modeling on long dependencies.

Human Activity Recognition with Convolutional Neural Networks (CNN) [24], recurrent neural network RNN [25], and other deep learning techniques still use wearable sensors or body-worn sensors and can only detect single and straightforward activity. Whoever detects some complex activity suffers from accuracy and complexity. If they use external sensors also, they only focus on just the single activity recognition. Many researchers also used classification algorithms such as skip chain conditional random field (SCCRF) [7], Decision Tree [26], Hidden Markov Model (HMM) [27], Conditional Random Field (CRF) [28]. Still, these approaches get rid of temporal differences between events. In this work, we focus most of our analysis on concurrent and interleaving activity using the extended LSTM and CRF due to their widespread popularity and a proven track record. The growing interest in LSTM-RNN enhanced many technical applications such as language modeling, handwriting recognition, and generation [29], machine translation [30], speech recognition [31], video analysis [32], and image captioning [33] least compare to activity recognition. SCCRF were also have been used for natural language processing and other specific task detection problem like behavior recognition [34]. Meanwhile, an abundance of work, architecture, and model has been developed, but relatively less attention has been paid to understand the properties of its concurrency and interleaved representations and predictions.

\section{Materials and Method}

Figure 2 explains the overall system (process) of activity recognition. While recognizing activity phenomena, our interest is in recognizing complex activity such as concurrent and interleaved activity. The activities done at the same time is a concurrent activity, such as talking with friends while walking or listening to music while sleeping. Both the activities must be recognized concurrently, as shown in Figure 2a. When one activity is paused to do another activity and continues the previous activity after finishing, that is the interleaved activity. That means some real-life activities are interleaved in nature, as shown in Figure 2b. For instance, if a bell rings at the door while watching TV, we pause watching TV and go to open the door, and we continue to watch TV. The activities always may not be concurrent and interleaved separately; it may occur in the combine, as shown in Figure 2c. Sleeping by listening to music can be paused while talking with friends on the 
phone and may continue sleeping by listening to music after talking is over. This paradigm is the most critical aspect of activity recognition but its always been avoid due to its complexity.

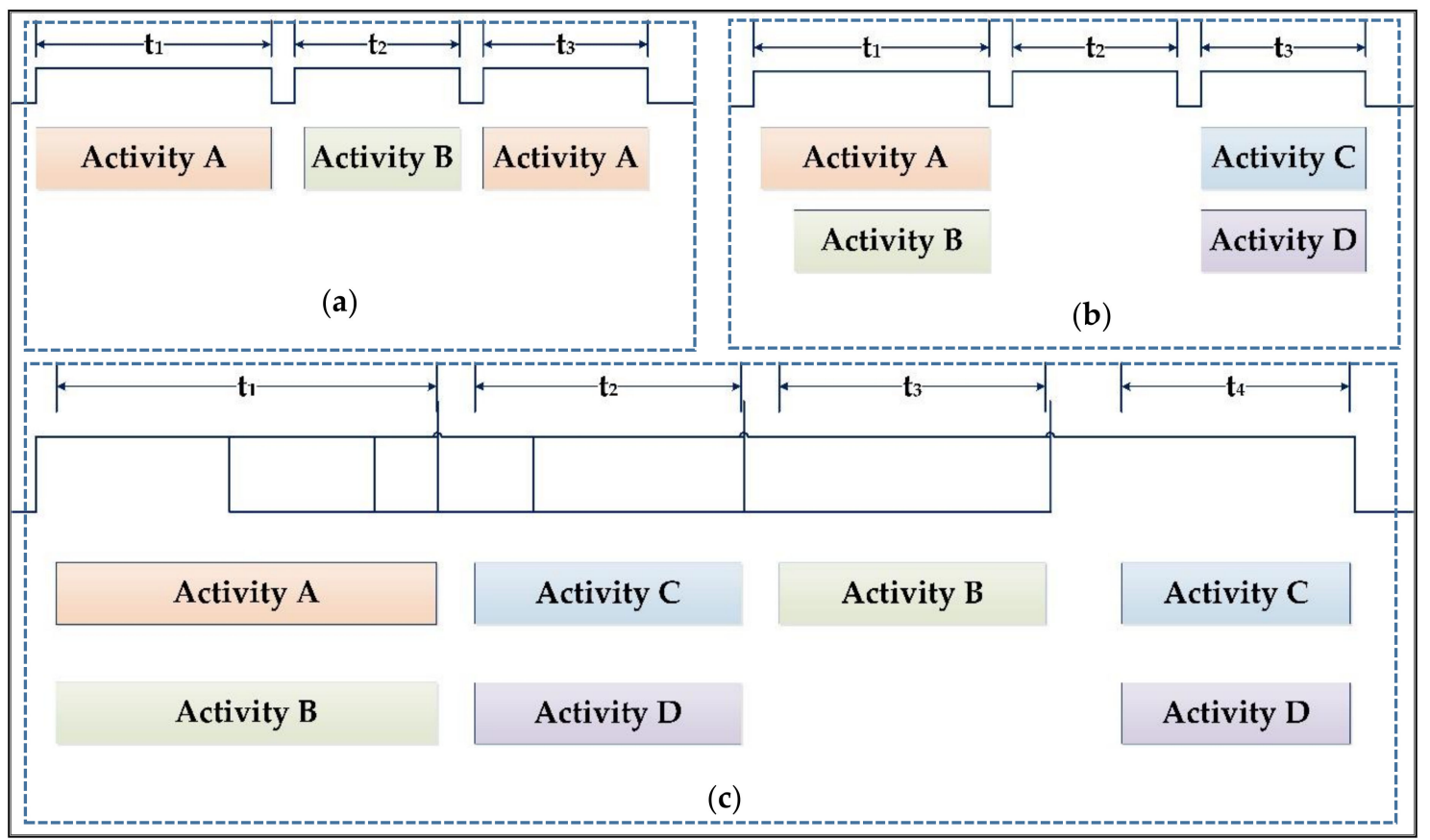

Figure 2. Pictorial view of (a) Interleaved Activity occurrence, (b) Concurrent Activity occurrence, (c) Concurrent and Interleaved Activity.

\subsection{Long-Short Term Memory (LSTM)}

Recurrent Neural Networks (RNNs) make the output use the previously memorized information extracted from the past data to the neurons. This specific ability of RNNs of the neurons to find patterns with long-term dependencies. However, the problem of vanishing gradient may appear. These happened when the error function derivatives concerning the network weights become either very large or close to zero during the training phase effects the RNNs. The proposed Long-Short-Term-Memory (LSTM) cell addresses this issue. LSTM designed to memorize the information over time by storing it in an internal memory called a cell and update output or erase their internal state depending on their input and the state at the previous time step. LSTM networks capture temporal dependencies in diversified application fields such as image captioning, automatic translation, or video-based activity recognition. The LSTM architecture comprises memory cells with self-connections for storing high temporal dependencies, as shown in Figure 3. They contain the gates to control the flow of information. The memory cell consists of a node with a fixed weight with a self-connect loop edge so that the gradient can propagate across many time steps without vanishing. An input gate controls the input activation data into memory cells; the primary function of this gate is to write on memory cells. An output gate control and read output data flow of cell activations. Forget gate decides which data to be forgotten or resetting the cell's memory.

\subsection{Bi-Directional LSTM}

The baseline LSTM algorithm can recognize current activity based only on prior information. However, some vital information about the activity may not be adequately extracted by the cell if it processes only one direction. The principal feature in bidirectional LSTM is that it follows information and current output related to former details. Bidirectional LSTM is a design of two LSTM cells, and the output is determined together, as shown in Figure 4. 


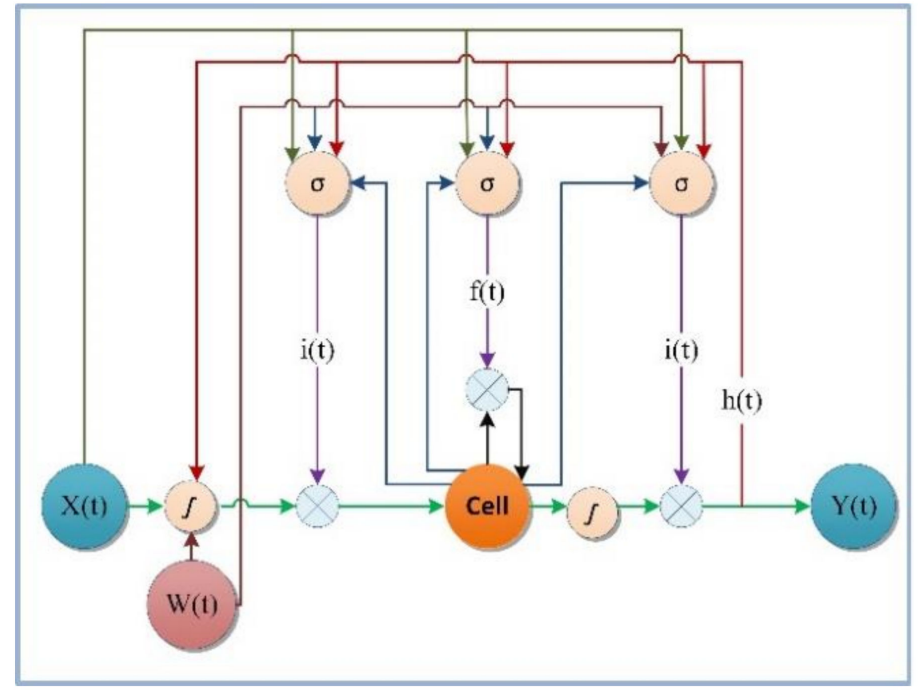

Figure 3. LSTM Architecture.

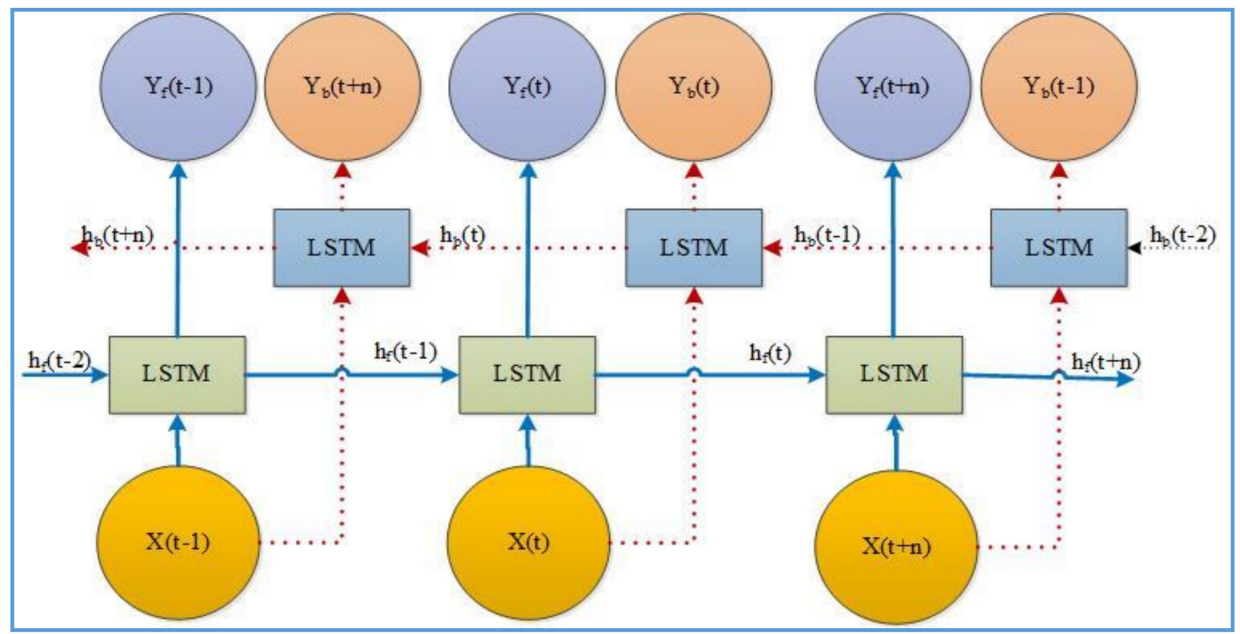

Figure 4. Bi-directional LSTM architecture.

The LSTM architecture also contains peephole in internal cells to gates in the same cell to learn the outputs' precise timing. The multiplicative equation controls each cell and gate to the forward propagation:

$$
\begin{gathered}
i(t)=\sigma\left(w_{x i} x(t)+w_{h i} h_{f}(t-1)+w_{c i} c(t-1)+b_{i}\right) \\
f(t)=\sigma\left(w_{x f} x(t)+w_{h f} h_{f}(t-1)+w_{c f} c(t-1)+b_{f}\right) \\
o(t)=\sigma\left(w_{x o} x(t)+w_{h o} h_{f}(t-1)+w_{c o} c(t-1)+b_{o}\right) \\
c(t)=f_{t} c(t-1)+i(t) \tanh _{f}\left(w_{x c} x(t)+w_{h c} h(t-1)+b_{c}\right) \\
h_{f}(t)=o(t) \tanh _{f} c(t)
\end{gathered}
$$

$x(t)$ is the input sequence and $w_{x i}, w_{x f}$ and $w_{x o}$ being the input weight matrix of $i(t)$ an input gate, $f(t)$ a forget gate and $o(t)$ an output gate, having the same vector size as the vector of hidden value.

The $c$ represents the memory cell, and $\mathrm{b}$ represents the bias of the respective gate and cell. The hidden state $h(t)$ processes input $x(t)$ and thus yields output $y(t)$ at time $t$ by unrolling LSTM cells, as shown in Figure 4. $\sigma$; known as the logistic sigmoid function or the sigmoid activation function that 
limits the value in between $[0,1]$ and given as $\sigma(x)=\frac{1}{1+e^{-x}}$. tanh; Hyperbolic tangent activation function. The forward propagation executes input $x(t)$ from left to right with a hidden layer of $h_{f}$, and backward propagation executes input from right to left with the hidden layer of $h_{b}$ as according:

$$
\begin{gathered}
\delta_{i}(t)=\sigma\left(w_{y i} x(t)+w_{h i} h_{b}(t+1)+w_{c i} c(t+1)+b_{i}\right) \\
\delta_{\mathrm{f}}(\mathbf{t})=\sigma\left(\mathrm{w}_{\mathrm{yf}} \mathrm{x}(\mathrm{t})+\mathrm{w}_{\mathrm{hf}} \mathrm{h}_{\mathrm{b}}(\mathrm{t}+1)+\mathrm{w}_{\mathrm{cf}} \mathrm{c}(\mathrm{t}+1)+\mathrm{b}_{\mathrm{f}}\right) \\
\delta_{\mathrm{o}}(\mathrm{t})=\sigma\left(\mathrm{w}_{\mathrm{yo}} \mathrm{x}(\mathrm{t})+\mathrm{w}_{\mathrm{ho}} \mathrm{h}_{\mathrm{b}}(\mathrm{t}+1)+\mathrm{w}_{\mathrm{co}} \mathrm{c}(\mathbf{t}+1)+\mathrm{b}_{\mathrm{o}}\right) \\
\delta_{c}(t)=f_{t} \delta_{c}(t+1)+\delta_{i}(t) \tanh _{b}\left(w_{y c} x(t)+w_{h c} h_{b}(t+1)+b_{c}\right) \\
h_{b}(t)=\delta_{o}(t) \tanh _{b} \delta_{c}(t)
\end{gathered}
$$

Therefore, in this work, we focused on recognizing concurrent and interleaving activity and its analysis. Meanwhile, an abundance of research work modifies or extends LSTM architecture to understand the properties of its complex representations and predictions. [35] Recently evaluated a comprehensive study of LSTM components. The most leading deep LSTM networks for the HAR method for human activity recognition using wearable sensing approaches exploit the contextual information and adopt the temporal dependencies in variable-length input data [36].

\subsection{Skip Chain CRFs}

The SCCRFs can represent dependencies between distant nodes so that similar activity can be detected. The SCCRF [37] can contain information from the activity of both times. Because the activity of one time is specific, it can impact the activity of the other time. We have to choose an identical activity that belongs to the same class or have a small edit distance, as shown in Figure 5.

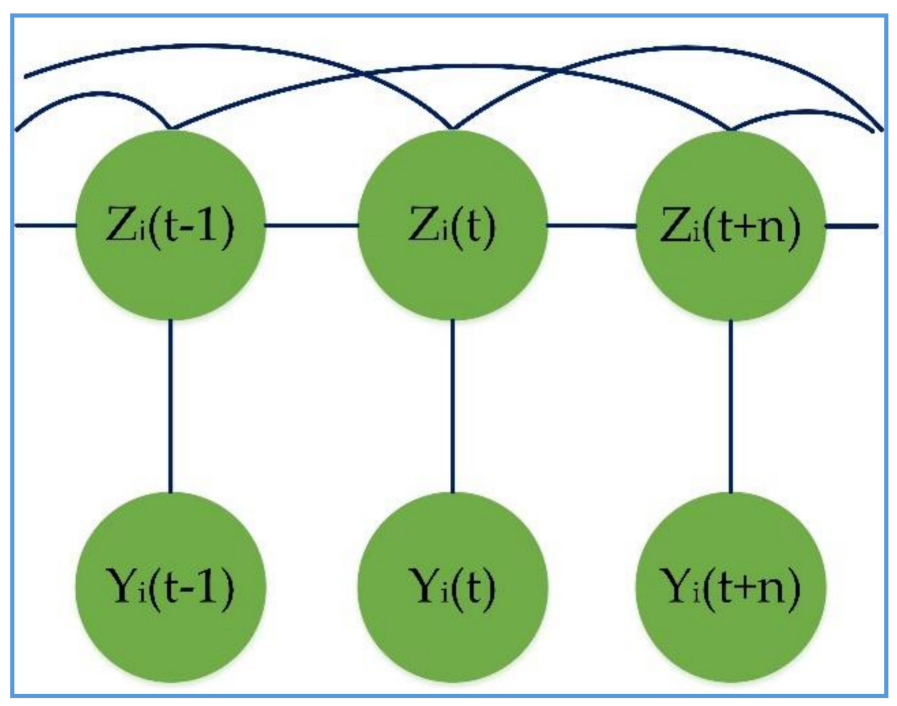

Figure 5. SCCRF Representation.

However, we connect all possible activity because it requires excessive programming algorithm. Therefore we are only concern about the edges (activity) that appeared to be active or not at time slice $t$. Given observation sequence $Y$, let $Z$ be all pairs of active activities connected with skip edges. Thus the probability of activity sequence $Z$ given an observation activity $Y$ can be written as;

$$
\mathrm{P}(\mathrm{z}, \mathrm{y})=\frac{1}{\mathrm{~S}(\mathrm{y})} \prod_{\mathrm{t}=1}^{\mathrm{n}} \Phi\left(\mathrm{z}_{\mathrm{t}}, \mathrm{z}_{\mathrm{t}-1}, \mathrm{y}, \mathrm{t}\right) \prod_{(\sqcap \sqsubseteq) \in \mathrm{I}} \Psi\left(\mathrm{z}_{\sqcap}, \mathrm{z}_{\sqsubseteq}, \mathrm{y}, \sqcap, \sqsubseteq\right)
$$


$\Phi$ is the values over linear-chain edges, and $\Psi$ is the potential factor over skip edges. $S(y)$ is a normalization factor where each of the potential factors is factorized by taking the log.

$$
\begin{gathered}
\Phi\left(\mathrm{z}_{\mathrm{t}}, \mathrm{z}_{\mathrm{t}-1}, \mathrm{y}, \mathrm{t}\right)=\exp \sum_{\mathrm{k}} \lambda_{\mathrm{k}} \mathrm{f}_{\mathrm{k}}\left(\mathrm{z}_{\mathrm{t}}, \mathrm{z}_{\mathrm{t}-1}, \mathrm{y}, \mathrm{t}\right) \\
\Psi\left(\mathrm{z}_{\sqcap}, \mathrm{z}_{\sqsubseteq}, \mathrm{y}, \sqcap, \sqsubseteq\right)=\exp \sum_{\mathrm{k}} \lambda_{\mathrm{k}}^{\prime} \mathrm{f}_{\mathrm{k}}^{\prime}\left(\mathrm{z}_{\sqcap}, \mathrm{z}_{\sqsubseteq}, \mathrm{y}, \sqcap, \sqsubseteq\right)
\end{gathered}
$$

$\lambda_{k}$ and $\lambda_{k}^{\prime}$ are the parameters of the linear and skip chain template on which each of them factorizes according to a set of features $f_{k}$ or $f_{k}^{\prime}$ respectively. In skip-chain CRFs, exact inference $\mathrm{P}(\mathrm{z} \mid \mathrm{y})$ is much more difficult due to long loops and overlapping in the model. Instead, we approach approximate inference utilizing the belief propagation algorithm that works as the principle of the forward-backward algorithm for HMM. However, LPA passages information between the long-distance edges, emitting probabilistic information from distant sequences.

For the given training data, we estimate the parameter $\lambda_{k}$ by maximizing the log-likelihood after calculating the partial derivative and optimization technique

$$
\frac{\partial \mathrm{P}(\mathrm{z} \mid \mathrm{y})}{\partial \lambda_{\mathrm{k}}}=\frac{\partial \mathcal{L}}{\partial \lambda_{\mathrm{k}}}=\frac{\lambda_{\mathrm{k}}}{\sigma^{2}}
$$

where $\mathcal{L} \log$-likelihood and $\sigma^{2}$ is the covariance as assuming that the long-distance parameter is similar, therefore replacing $\lambda_{k}^{\prime}$ by $\lambda_{k}$ and $f_{k}^{\prime}$ by $f_{k}$.

\subsection{Proposed Method}

The schematic architecture in Figure 6 demonstrates complex activity recognition. Our proposed approach has the two-phase structure in which the first phase processes under the bi-directional LSTM theorem, and the second phase implement a theory of Skip-Chain CRF (SCCRF). It performs a direct mapping from raw multi-modal sensor inputs to activity classification and recognition. It recognizes the label of performed activity during a specific time window. The input is an equally spaced discrete sequence of samples $\left(x_{t-2}, x_{t-1} x_{t}, x_{t+1} \ldots, x_{t+n}\right)$ where each data point $x_{t}$, is a set of individual observed samples by sensors at time $t$. The extracted samples are segmented into windows and fed to the BLSTM-based model. The model outputs a sequence of scores representing activity predictions. The first phase represents the detection of concurrent activity; second, interleaved activity. The bi-direction LSTM network contains two parallel LSTM tracks; forward and backward propagations for exploiting data from past and future of a specific time step to recognize the parallel activity. The output of the bi-directional LSTM determines the concurrency of the recognized activity. The same output is feedforward to recognize an interleaved condition of activity using the Skip-Chain Conditional Random Field (SCCRF). The model outputs a sequence of activity $\left(\mathrm{y}_{\mathrm{t}-2}, \mathrm{y}_{\mathrm{t}-1} \mathrm{y}_{\mathrm{t}}, \mathrm{y}_{\mathrm{t}+1} \ldots, \mathrm{y}_{\mathrm{t}+\mathrm{n}}\right)$ in which there is an activity prediction for each time step $\left(\mathrm{z}_{\mathrm{t}-2}, \mathrm{z}_{\mathrm{t}-1}, \mathrm{z}_{\mathrm{t}}, \mathrm{z}_{\mathrm{t}+1} \ldots, \mathrm{z}_{\mathrm{t}+\mathrm{n}}\right)$. SCCRF finds the probability of activity that has been paused to perform a crucial uncertain task. The SCCRF is very reliable and efficient in finding the possibility of a similar activity performed in activity sequences. A schematic diagram in Figure 7 gives concepts of an overall system architecture of the proposed. In the first phase, the input sensor data or sampled data is transformed through a sliding window to produce data; this procedure is regarded as data preprocessing. Prepared data is inserted into the first layer Bi-LSTM is processed until it adapts its internal state. An passed into another Bi-LSTM layer where we applied the dropout to drop the unused neurons. The obtained result is normalized and fed into a fully connected layer, which is also known as the coding layer and hence recognizes the concurrent activity. In the second phase, the data's sequence and the data from the fully connected layer are inserted into the clique template. Clique template makes surmise on the structure of data by defining the configuration of the cliques. A clique is a set of interdependent data. And finally, the SCCRF model 
is used to recognize the interleaved activity. Our concern target is to develop a reliable algorithm implementing both the machine learning and deep learning into a single frame (Algorithm 1).

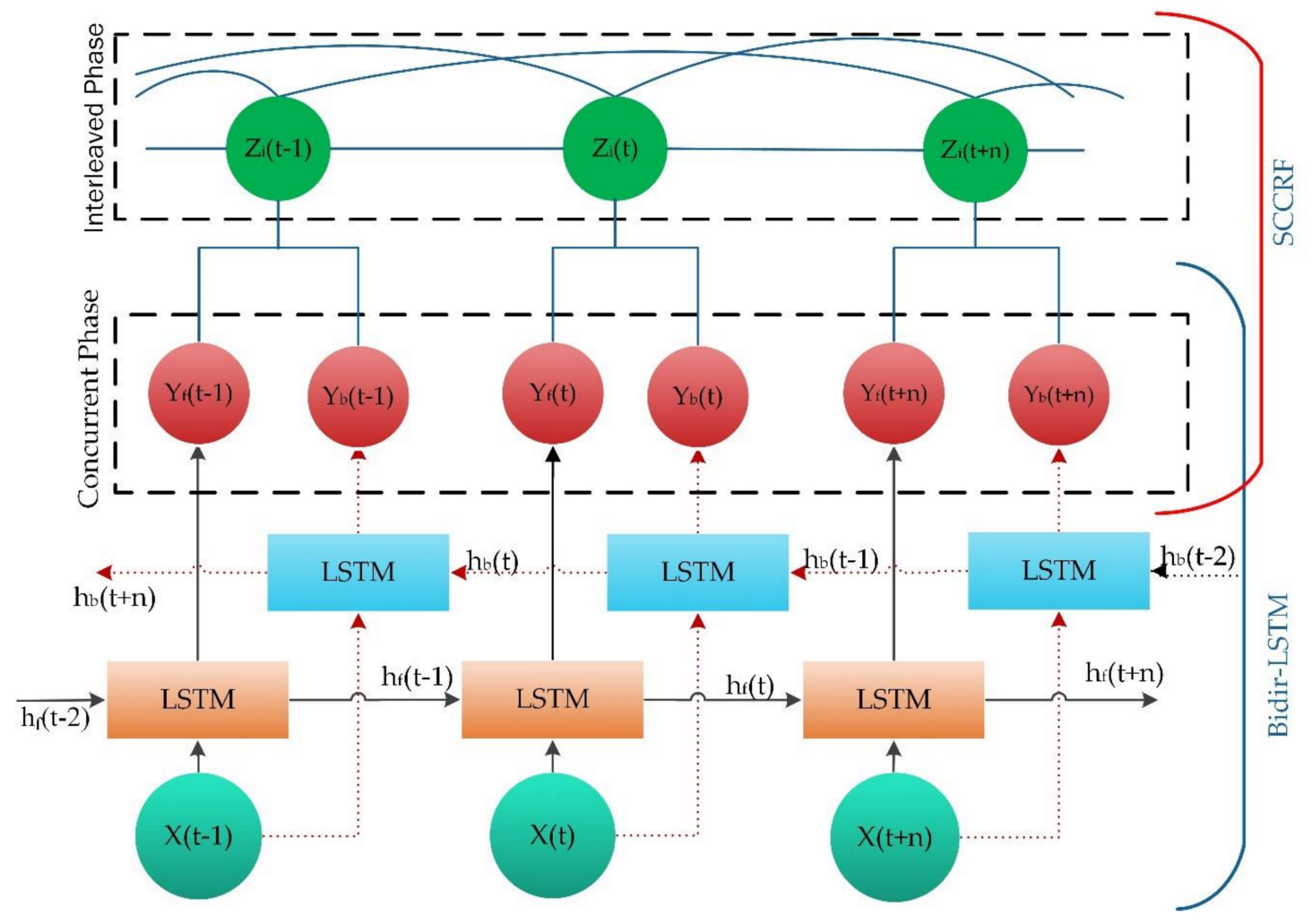

Figure 6. Proposed schematic architecture.

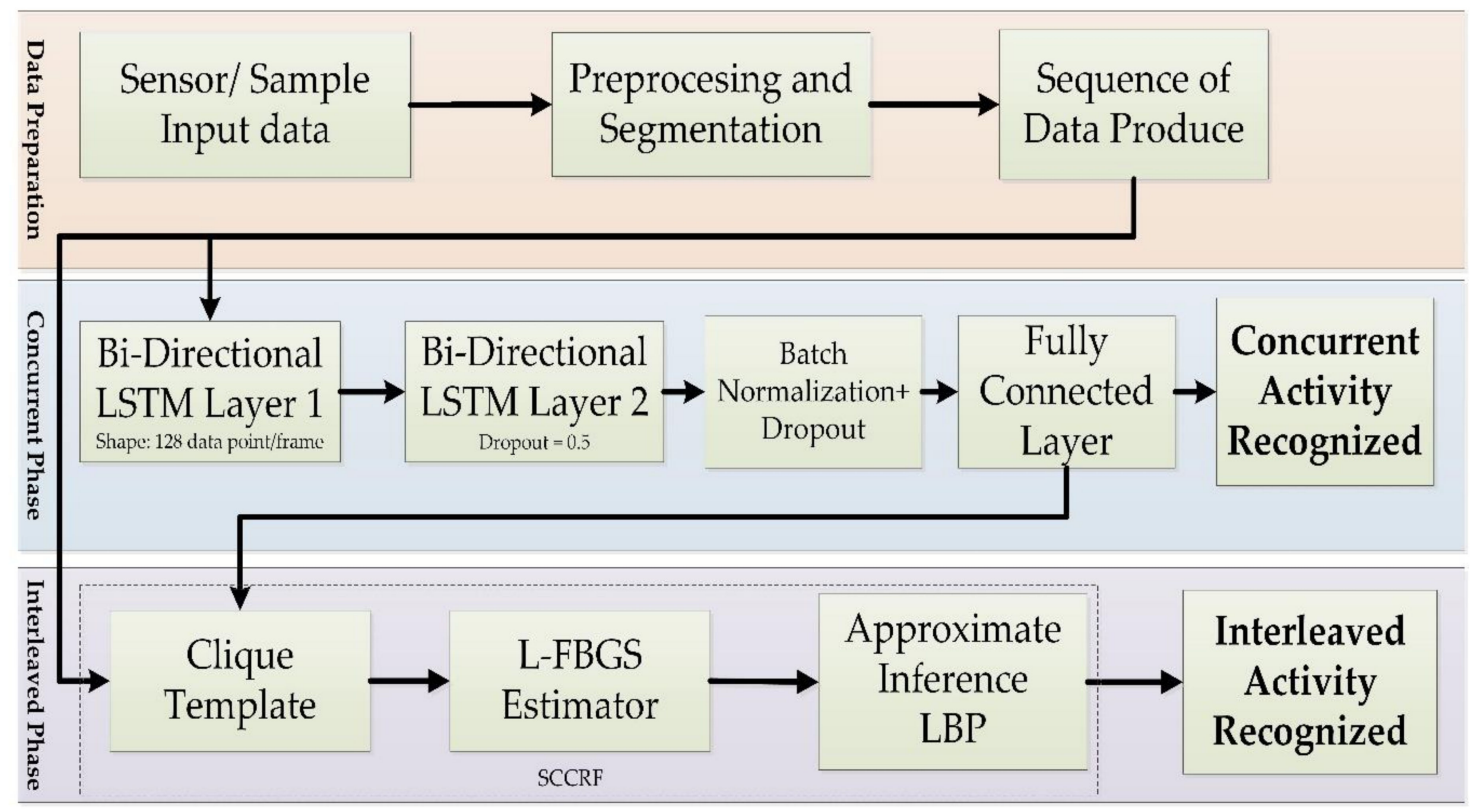

Figure 7. Overall system structure of the proposed approach. 


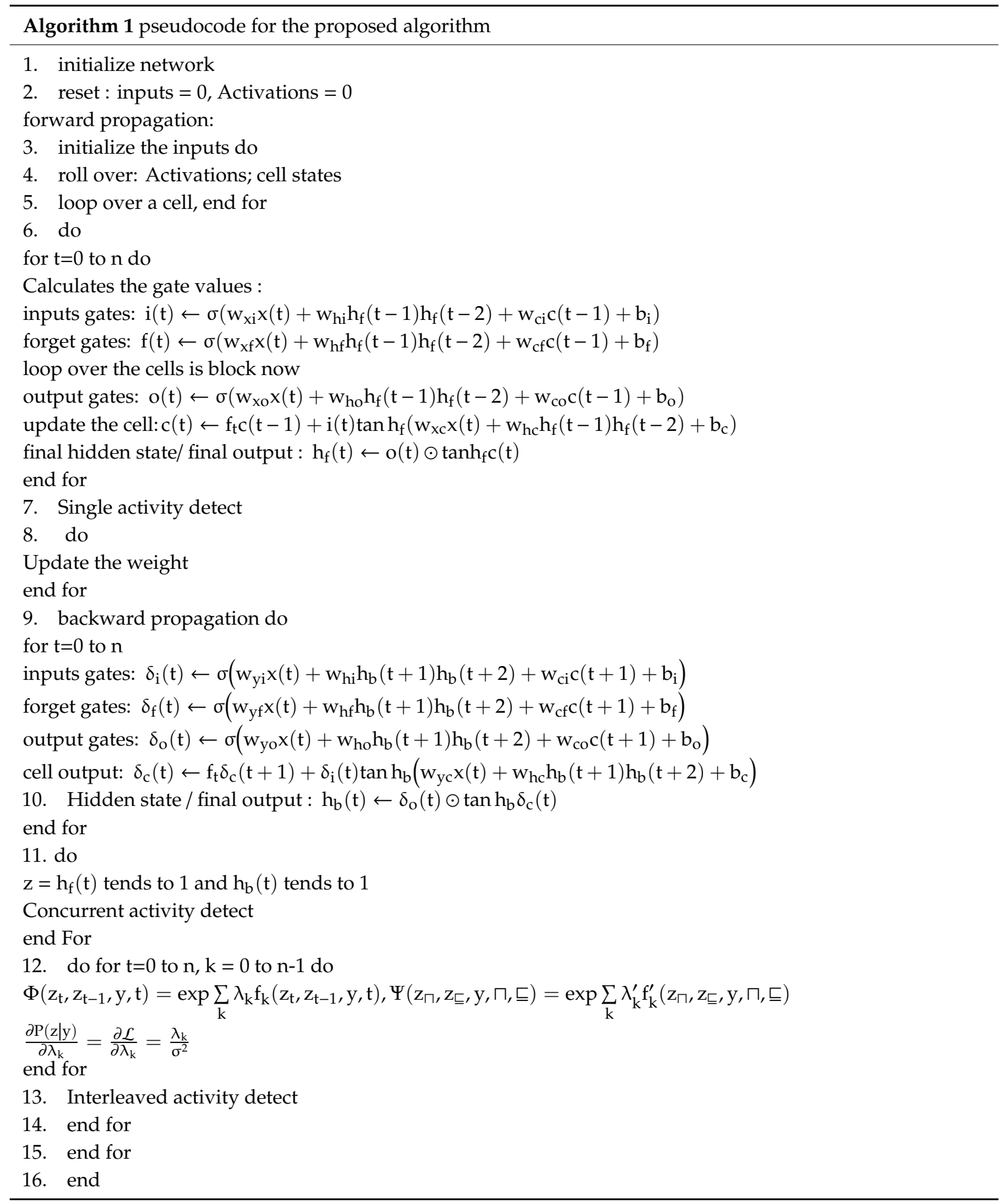

\section{Experimental Configuration}

Our primary target is to recognize the complex activity using our novel approach. We experiment, evaluate, and analyze our proposed method using publically available datasets named; Kasteren HouseB from Kasteren [38] and Kyoto 3 from CASAS [39]. The pseudocode for the proposed approach is shown in Algorithm 1. Then we compare the results with other HAR methods.

\subsection{Benchmark Datasets}

Human activities are unpredictable and periodic. There are numerous datasets available. Mostly, concerns with simple activity with low instances. Furthermore, the sensors used are body-worn. The proposed method focused on using external sensors rather than body-worn. Human activities 
offered a large Null class that is more challenging to identify the start and endpoint of data and classify it. In more recognition, we ignore the Null class, as including the null class may lead to an overestimation of the recognition. Table 1 shows an overview of the datasets. The chosen datasets count the limitation of sharing features among the activity instances of different groups, the number of inhabitants in each home, their performance of the same activity in different ways, and the availability of fewer data for learning. The twenty-three and seventy-six different heterogeneous sensors collect the data in Kasteren house-B and Kyoto, respectively. Among 76 sensors, 51 sensors were used to detect motion sensors and five sensors to detect the cooking item. Twelve sensors as a cabinet sensor, three sensors as a temperature sensor, and another sensor as a medicine container sensor, pot sensor, phone book sensor, water sensor, burner sensor, and phone sensor. For the Kasteren House, sensors like RFID (Radio Frequency Identification), Pressure sensor, mercury contacts, passive infrared-PIR, float sensors, reed switches, and temperature sensors are deployed to capture the data. The schematic sensor layout of the dataset is shown in Figure 8. There are 135 and 178 activity instances from which the 13 and 8 activity will be recognized in a concurrent and interleaved fashion. The sensors represent the change of state according to the action of users.

Table 1. Overview of datasets used in the evaluation of the proposed method.

\begin{tabular}{|c|c|c|}
\hline Description & Kasteren House-B & Kyoto 3 \\
\hline Setting & Apartment & Apartment \\
\hline Rooms & 2 & 4 \\
\hline Senors & 23 & 76 \\
\hline Activities & 13 & 8 \\
\hline Residents & 1 & 4 \\
\hline Period & $14 \mathrm{~d}$ & $15 \mathrm{~d}$ \\
\hline Instances & 135 & 178 \\
\hline Activities Performed & $\begin{array}{c}\text { Breakfast, Brushing Teeth, Dinner, } \\
\text { Drinking, Dressing, Leaving } \\
\text { House, Others, Preparing } \\
\text { Breakfast, Preparing Dinner, } \\
\text { Sleeping, Showering, Toileting, } \\
\text { Using Dishwasher }\end{array}$ & $\begin{array}{l}\text { Fill Medication Dispenser, Wash } \\
\text { DVD, Water Plants, Answer the } \\
\text { Phone, Prepare Birthday Card, } \\
\text { Prepare Soup, Clean, Choose Outfit }\end{array}$ \\
\hline
\end{tabular}

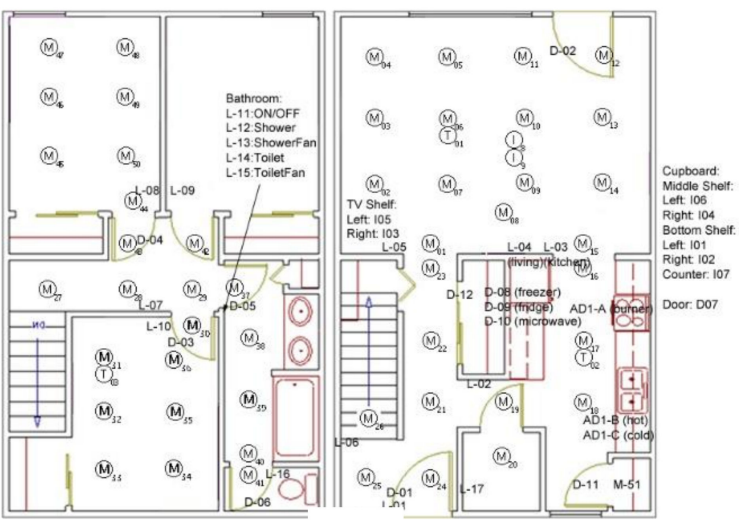

(a)

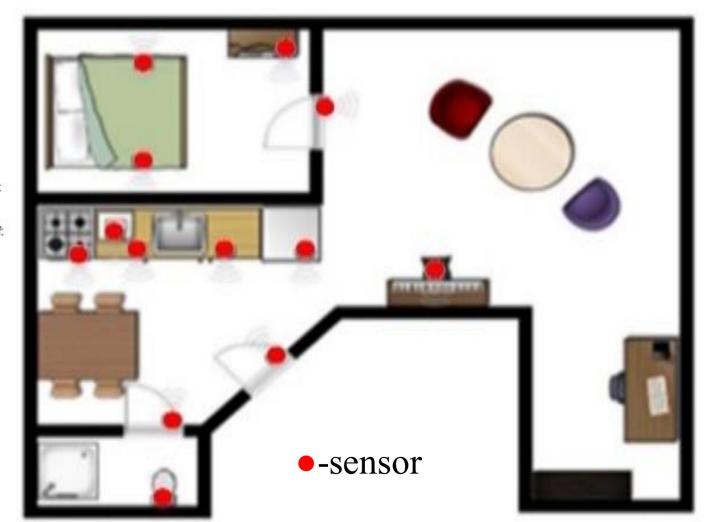

(b)

Figure 8. Sensor layout for (a) Kyoto 3 Dataset (b) Kasteren House B Dataset.

\subsection{Parameter Setup and Training}

The datasets were segmented into different window ranges, as shown in Figure 9. The maximum ranges of windows depending on the activities performed by the human and the sampling rate. In this experiment, we choose the best ranges window that gave better results by performing various samples. In the uncertain and real-time scenario, varied window length plays a perfect role 
rather than fixed scales because the network has to wait to complete a given range to proceed further. When the mean cross-entropy of actual outputs and predicted outputs are measured as updated cost function, then a cost function must be minimized by randomly initializing the approach's weight parameters. The actual output is given in the datasets and indicates real output activity for segmented windows. The predicted outputs contain every output generated by our method.

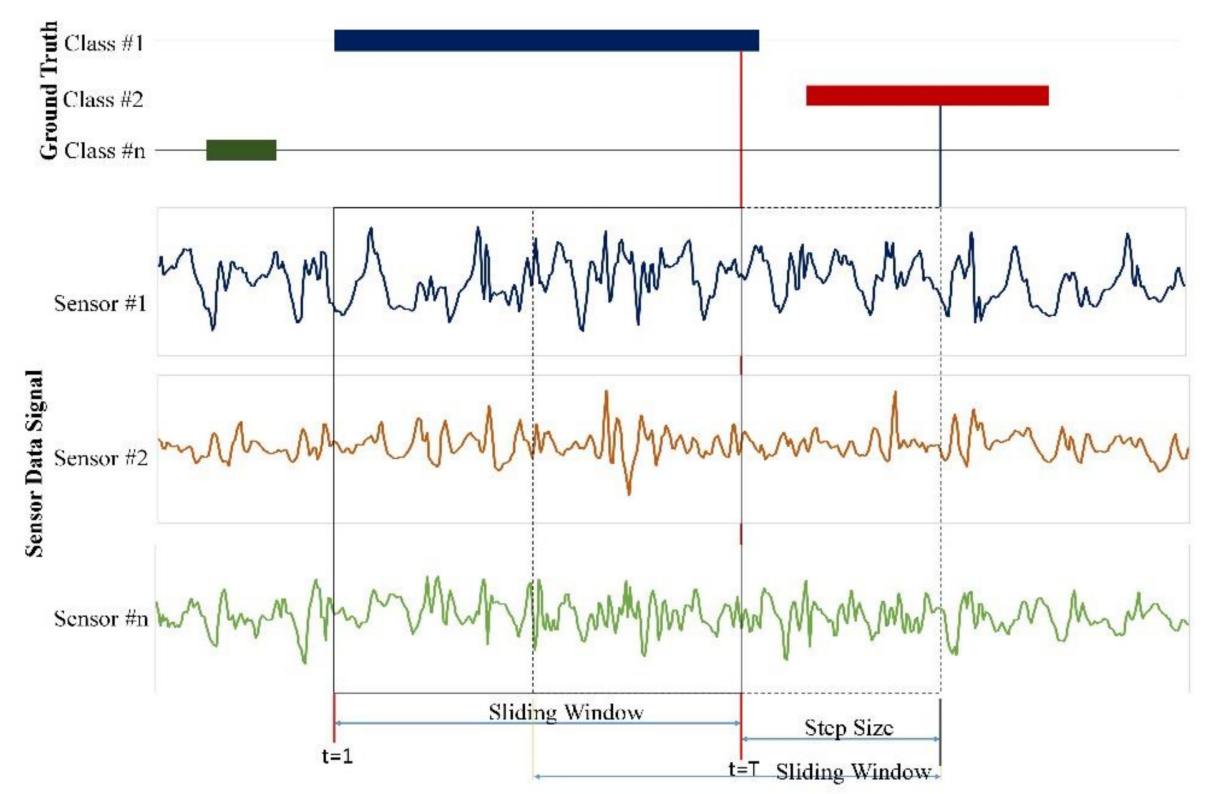

Figure 9. Sequence labeling after segmentation of data with a sliding window.

During the training iteration, the dropping at nodes in the network is tuned and represents units of the drop by using hyper-parameter. This dropping technique narrowed the accuracy gap between training and testing costs, which means dropping technique over through overfitting problems. The main reason behind using dropout is to disable the neurons with low probability, which improves the model generalization. The proposed method is implemented in the TensorFlow_gpu1.13.1 library. The computer taken for testing is best suitable to run our algorithm with i7 CPU with 16GB RAM with GTX titan GPU with CUDA 9.0 and used the cuDDN 7.0 library. CUDA is a platform that eases for parallel programming and also an interface for application programming. CuDDN is a GPU facilitated library of primitives for deep neural networks. $80 \%$ of the data are the training set, and the remaining $20 \%$ of data is for the test set. We record the Kasteren and Kyoto datasets. The training and testing use a sliding window of fixed length to segment the data, but data inputs may be variable length windows in real-time data scenarios. Following the preferred dataset experimental setup, the proposed approach depicts $500 \mathrm{~ms}$ lengthen window size with a $250 \mathrm{~ms}$ step size. The number of instances acquired after using this sliding window arrangement per the depicted dataset. The class corresponds to the observed sensor during that interval is associated with each segment. The sequence's activity as the label at $t=n$, i.e., the last sample's label in the sliding window of length ' $n$ '. A sliding window segments the sensor signals. The activity class within each activity sequence is the ground truth label annotated at the sample $\mathrm{n}$ of that window.

The best-suited hyper-parameters are tuned, as shown in Table 2, and achieve the possible optimal performance. We train the model with a learning rate of 0.005 , and 100 batches of each epoch for the Kasteren House and the learning starts at 0.001 . The training is carried out for more than 10,000 epochs and stops on stable outputs. The Adam optimizer is an adaptive moment estimation that obtains independent adaptive learning rates for different parameters making them more stable for the Bi-LSTM and Quasi-Newton optimizer by the SCCRF. The dimension based on the input is set to 128 . The gradient clipping is set to 5 , whereas this parameter scaled-down the exceed 
gradient threshold to match the normalization. Also, In this paper, the SCCRF model uses the Limited-Memory Broyden-Fletcher-Gold-Shano (L-BFGS) algorithm to estimate the parameter, and the Loopy Belief Propagation (LBP) algorithm to perform the approximate inference. We used the 10-fold cross-validation technique to evaluate models. This may also help to estimate the capabilities of a model on unseen data.

Table 2. Hyperparameter Settings.

\begin{tabular}{cc}
\hline Hyperprameters & Values \\
\hline Time Steps of input & 128 \\
Dropout Rate & 0.5 \\
Initial Learning Rate & 0.001 \\
Learning Rates & 0.005 \\
Optimizer (Bi-LSTM) & Adam \\
Batch Size & 100 \\
Gradient Clipping & 5 \\
Skin-chain parameter $\theta$ & $\sum_{k} \lambda_{k}$ \\
SC Optimizer & Quasi-Newton \\
Epochs & 10000 \\
\hline
\end{tabular}

\subsection{Analysis Metrics}

We define the evaluation terminologies as precision, recall, F-score, and accuracy. F-score is the harmonic mean of precision and recall. These terminologies are measured using true positive (TP), false positive (FP), and false-negative (FN) through the confusion matrix.

$$
\begin{gathered}
\text { Precision : } \frac{1}{N}\left(\sum_{k=1}^{N} \frac{T P_{k}}{T P_{k}+F P_{k}} \times 100\right) \\
\text { Recall }: \frac{1}{N}\left(\sum_{k=1}^{N} \frac{T P_{k}}{T P_{k}+F N_{k}} \times 100\right) \\
\text { F- score }: \frac{2 \times \text { Precision } \times \text { Recall }}{\text { Precision }+ \text { Recall }} \\
\text { Accuracy }=\frac{t p+t n}{t p+t n+f p+f n} \times 100
\end{gathered}
$$

Each dataset is categorized into three-set: a training set, a validation set for optimizing the parameters, and a test set for end evaluation.

\section{Activity Recognition Performance Analysis}

In this section, the experimental results are explained and analyzed. All activities are located based on the dataset. The most frequently concurrent and interleaved activities are considered the presiding activities in the smart home environment. The analysis section is subdivided into concurrent activity recognition, interleaved activity recognition, and the recognition average. The overall recognition accuracy is then compared to the other state of art method; Hidden Markov model-Skip Chain Conditional Random field (HMM-SCCRF) [7], Long-Short Term Memory-Skip Chain Conditional Random field (LSTM-SCCRF) [40], and Bi-directional Long-Short Term Memory- Conditional Random field (Bi-LSTM-CRF) [41].

\subsection{Concurrent Activity Recognition Analysis}

Figure 10 represents a confusion matrix for Kyoto 3 for the recognition of concurrent activities. The proposed approach recognizes activities with satisfactory accuracy of more than $90 \%$. The filling 
medication dispenser and answering phone are concurrent with each other with an accuracy of $96 \%$ as both activity can be performed in parallel. Wash DVD, clean have $2 \%$ of their instances is shared as these activities share the same location. 3\% of watering plants is due to some kinds of error factors such as placement of motion sensor, frequent movement of inhabitants, and confusion in the kitchen location. The watering can be retrieved from the kitchen supply closet. Answering the phone is recognized concurrently with all the other activity with an accuracy of $94 \%, 97 \%, 94 \%, 93 \%, 95 \%$, and $95 \%$ to wash DVD, water plants, prepare birthday cards, prepare soup, clean, and choose outfit respectively. All the activities can be performed in a parallel manner while talking with high accuracy with ease. The Kyoto 3 data set's overall accuracy in recognizing the concurrent activities is more than 95\%, as shown in Figure 11, compared to other previously proposed approaches. There occurred a confusion between different activities trying to appear parallel with each other because of sharing the same location, platform, and some of the shared sensor values.

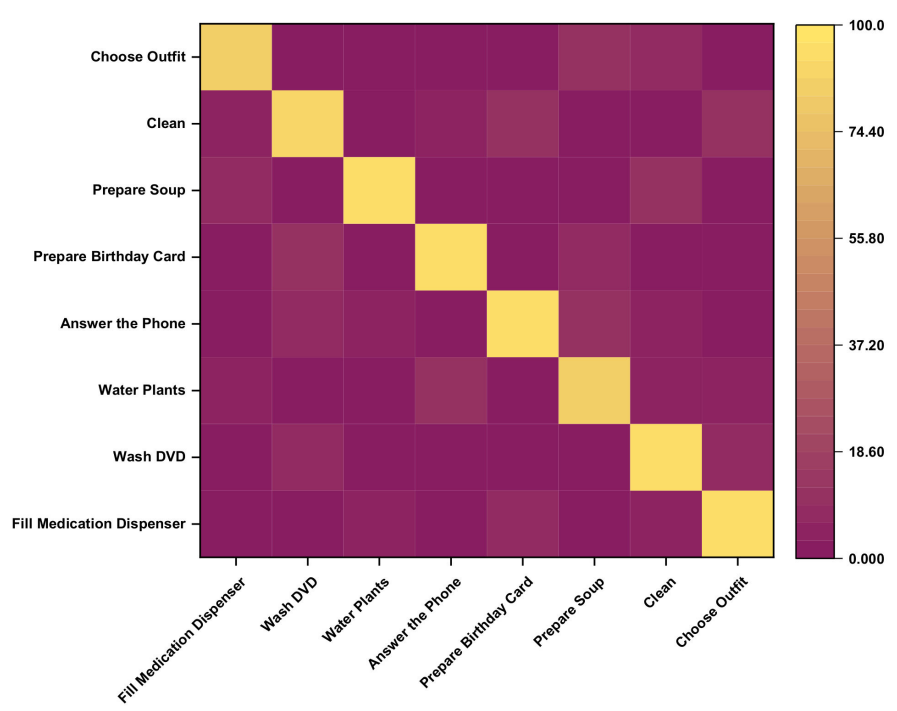

Figure 10. Confusion matrix for Kyoto 3.

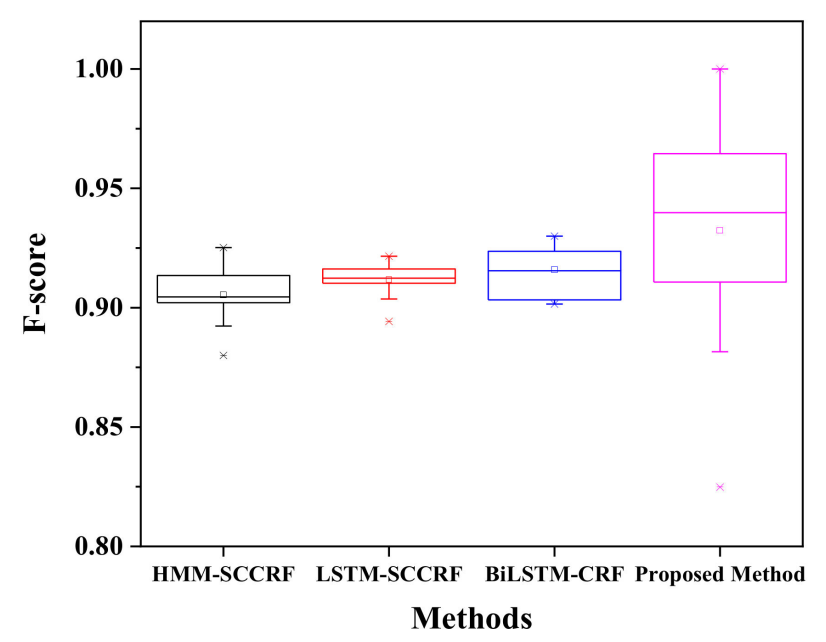

Figure 11. F-score comparison on Kyoto.

On the other hand, Figure 12 represents a confusion matrix of Kasteren-House-B to recognize the concurrent activity. While preparing breakfast, drinking, and eating can be done at the same time. The preparing breakfast activity is recognized concurrently to drinking activity with an accuracy of $97 \%$, and the same way, eating and drinking activities are detected concurrently with an accuracy of $93 \%$. 
$10 \%$ of error for dishwasher activity, $4 \%$ of error for other activity creates confusion. These activities are carried out in the same location at the kitchen. During this session location sensors, they are common and activated simultaneously time. Drinking activity has a high percentage of appearing B with an average accuracy of more than $93 \%$ to concurrently with other activities like breakfast $88 \%$, dinner $98 \%$, leaving the house $75 \%$. The only other activity does not have the concurrency because this activity is not well defined, and this is to reduce the error while recognizing the activity. The confusion matrix of two renowned datasets shows that recognizing concurrent activity using the proposed approaches and algorithm is quite impressive. However, a large number of datasets could help to get the actual and real recognition distribution. Kasteren and CASAS dataset have only a limited number of data and instances; therefore, it is a little bit easy to recognize, and accuracy may be high enough. Regarding analysis, our proposed method seems to be more reliable and effective than other compared models, as shown in Figure 13 on the same Kasteren house datasets.

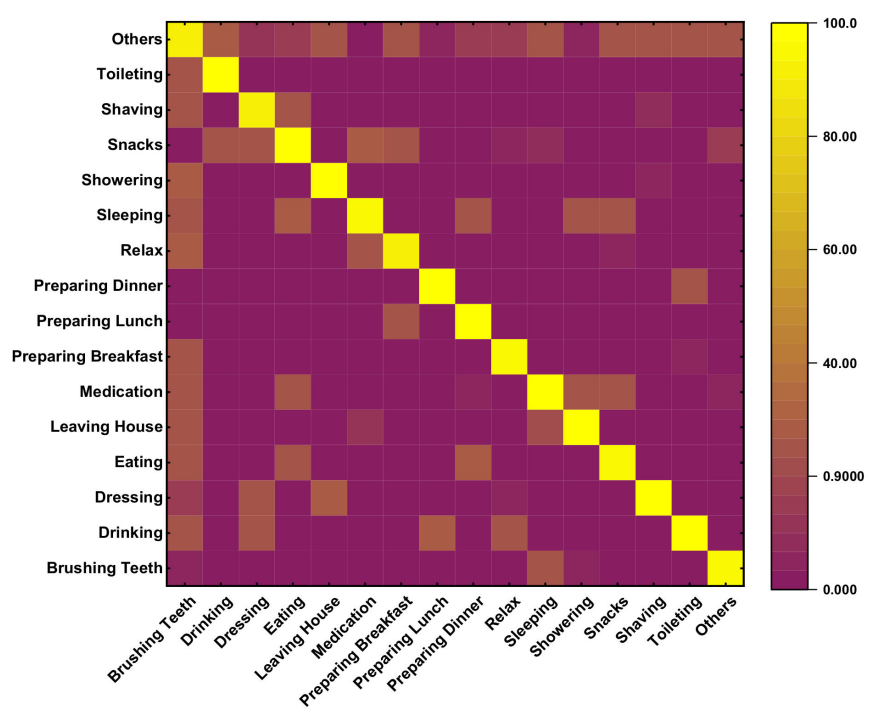

Figure 12. Confusion matrix for Kasteren-House-B.

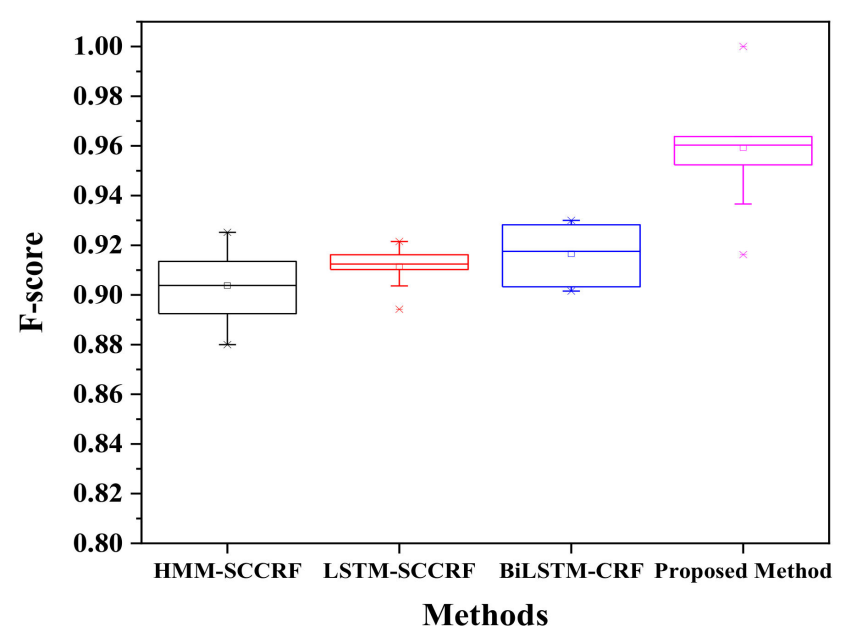

Figure 13. F-score comparison on KH-B.

\subsection{Interleaved Activity Recognition Analysis}

Interleaving activity is more complicated and challenging to recognize than concurrent activities; here, the activity is detected again after it has been performed a time before and reappeared at a specific time paused. The confusion matrix for Kastern House-B to detect the interleaved activity is shown 
in Table 3. The chances of recognizing breakfast activity after being paused are $93 \%$ while it has external instances error of $2 \%, 2.3 \%$, and $2.7 \%$ of dinner, breakfast, and others. Brushing Tooth can be re-recognized to $95 \%$, with an error of $5.2 \%$ each to toileting and showering; this is because the given activities share some information like location and sensor values. Sleeping has a $97 \%$ chance to recognize interleave fashion, but it has $6 \%$ confusion to the brushing tooth. It is due to the simultaneous occurrence of brushing teeth activity and sleeping. Activities like Dinner, Drinking, Dressing, Leaving House, Preparing Breakfast, Preparing Dinner, Showering, Toileting, Using Dishwasher, Others have the accuracy of $91 \%, 95 \%, 97 \%, 90 \%, 92 \%, 91 \%, 90 \%, 92 \%, 94 \%$ and $83 \%$ respectively. Therefore, the activity that appears other than the assigned activity is another activity. The proposed method recognizes the interleaving activities with an average accuracy of more than $92 \%$ with an f-score of 0.918 , as shown in Figure 14. Although the proposed method shows good results of the recognition of interleaving activity, there are so many confusion values that occurred. The main reason for this type of confusion is all because of sharing common location, common sensor values, the simultaneous occurrences of activity, and sharing the same platform.

Table 3. Confusion matrix for Kyoto 3.

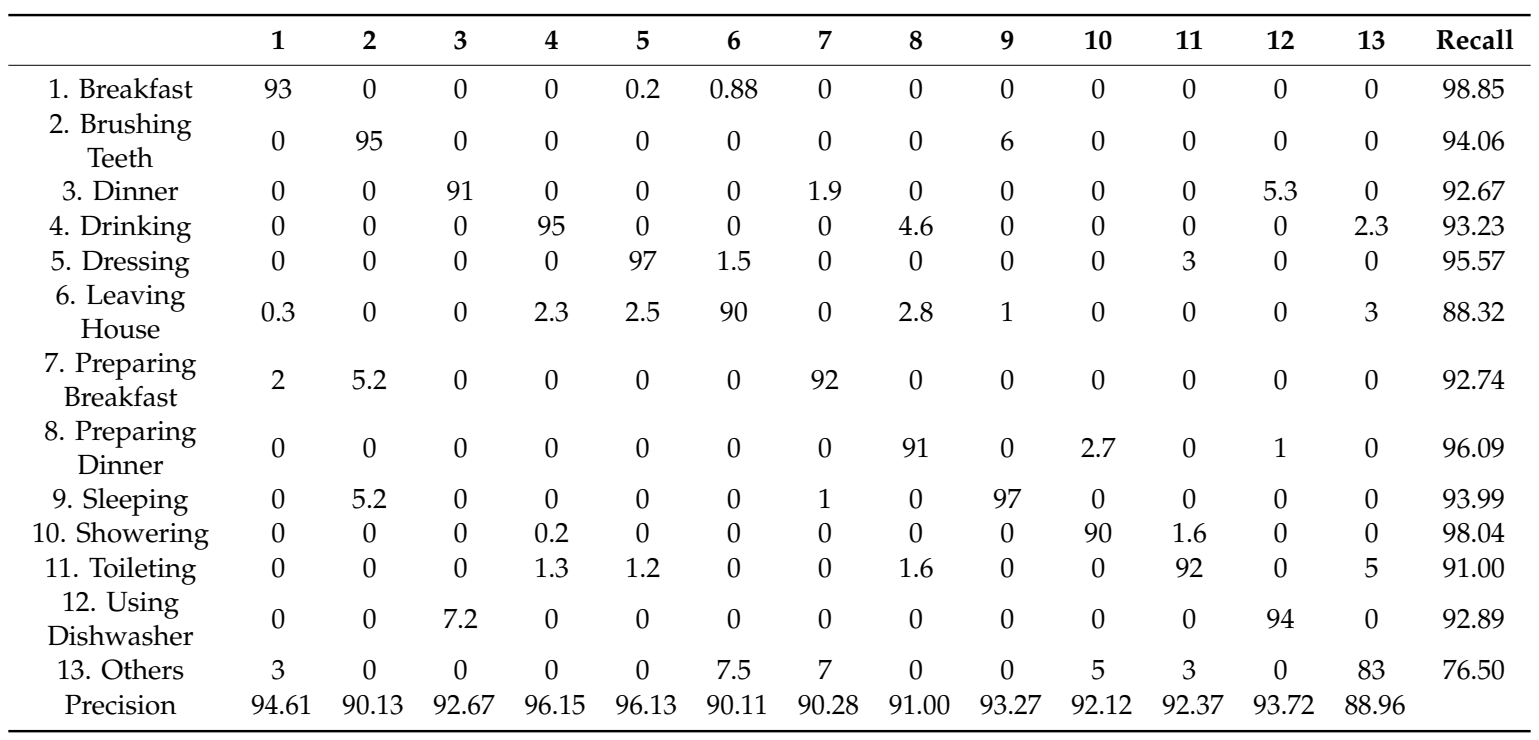

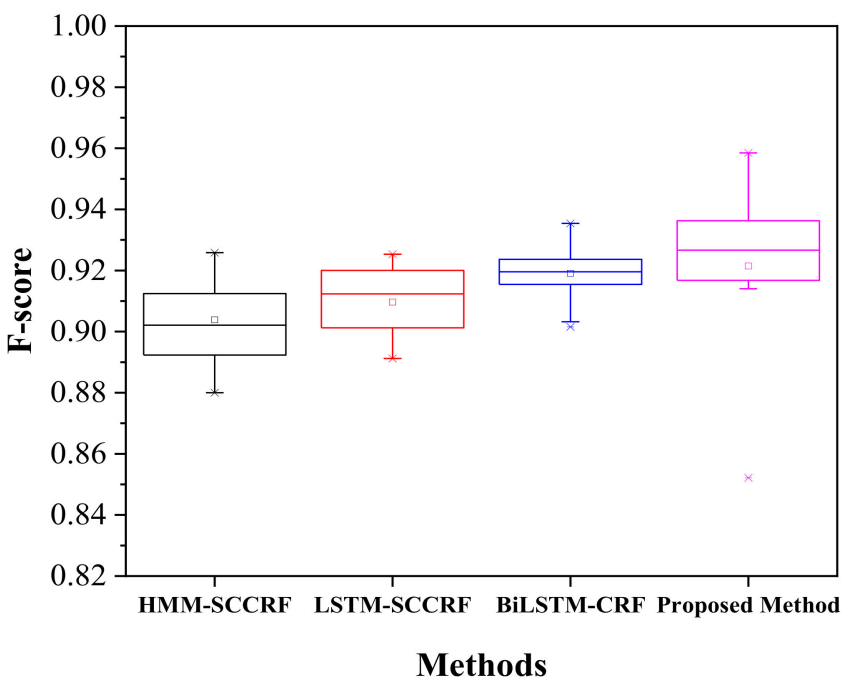

Figure 14. F-score comparison on KH-B. 
Table 4 represents the confusion matrix of recognition of interleaved activity for the Kyoto 3 dataset. The confusion matrix shows the average f-score of the recognition is above 0.95 , as given in Figure 14 , which shows the average accuracy is high to desire. As mention, due to limited data, the accuracy is pretty much in good shape. The recognition of the same activity, after pausing fill medication dispenser is about 93\%. Recognition of interleaved activity like Wash DVD, Water Plants, Answer the Phone, Prepare Birthday Card, Prepare Soup, Clean, Choose outfit is satisfactory with an average accuracy of $90 \%, 92 \%, 90 \%, 91 \%, 91 \%, 90 \%$, and $92 \%$ accordingly. The resultant recognition of interleaved activities by our proposed method outperforms existing approaches like HMM-SCCRF, LSTM-SCCRF, and Bi-LSTM-CRF. The resultant accuracy of Kyoto is more than 0.95 compared to other approaches, and algorithm 0.92 (HMM-SCCRF), 0.93 (LSTM-SCCRF), and 0.93 (Bi-LSTM-CRF) are as shown in Figure 15.

Table 4. Confusion matrix for Kyoto 3.

\begin{tabular}{llcccccccc}
\hline & $\mathbf{1}$ & $\mathbf{2}$ & $\mathbf{3}$ & $\mathbf{4}$ & $\mathbf{5}$ & $\mathbf{6}$ & $\mathbf{7}$ & $\mathbf{8}$ & $\mathbf{R e c a l l}$ \\
\hline 1. & Fill Medical on Dispenser & 93 & 1.3 & 2.7 & 0 & 0 & 1.6 & 0 & 0 \\
\hline 2. Wash DVD & 0 & 90 & 0 & 4 & 2 & 0 & 2 & 0 & 91.32 \\
\hline 3. Water Plants & 0 & 0 & 92 & 0 & 0 & 0 & 0 & 0 & 100.00 \\
\hline 4. Answer the Phone & 0.6 & 1.2 & 0 & 90 & 0 & 6 & 0 & 0 & 92.02 \\
\hline 5. Prepare Birthday Card & 0 & 3 & 0 & 0 & 91 & 0 & 0 & 2.3 & 94.50 \\
\hline 6. Prepare Soup & 5 & 0 & 0 & 2.3 & 3.2 & 91 & 0 & 0 & 89.66 \\
\hline 7. Clean & 2 & 0 & 5 & 0 & 1.2 & & 90 & 1 \\
\hline 8. Choose Outfit & 0 & 4 & 0 & 0 & 0 & 2 & 2 & 90.73 \\
\hline Precision & 92.45 & 90.45 & 92.28 & 93.46 & 93.43 & 90.46 & 95.74 & 96.54 \\
\hline
\end{tabular}

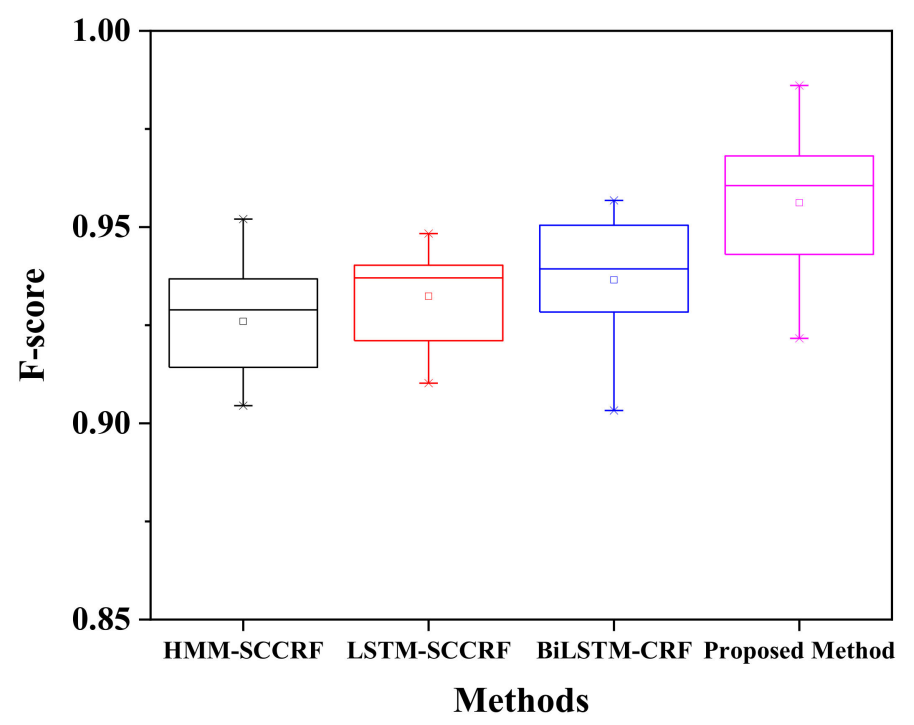

Figure 15. F-score comparison on Kyoto.

\section{Conclusions}

We adapt BiLSTM-SCCRF to establish a new approach to recognize concurrent and interleaved human activities within a single platform at a smart home. The proposed method recognizes activity in two phases. The first phase recognizes the concurrent activity using the bi-directional long short term memory (BiLSTM) extended version of RNN. The second phase is responsible for recognizing the interleaved activities using the skip-chain conditional random field (SCCRF) upgraded CRF version. By cascading these two phenomena, we can easily detect complex activity. This paper uses the two 
datasets to evaluate the proposed method's recognition performance with other recognition methods. In the evaluation process, F-score and accuracy are calculated from precision and recall. Also, Table 5 represents the mean and standard deviation by varying the Batch size hyperparameter on 10,000 epochs. Similarly, Table 6 shows the epochs parameter's result on sizing batch to 100, and the mean and standard deviation are calculated. The window size selection also plays a vital role in system accuracy; technically, $500 \mathrm{~ms}$ to $5000 \mathrm{~ms}$ window size extent will be relevant. The mean and standard deviation of accuracy and error using the 10-fold cross-validation is shown in Table 7. The average accuracy was found to be $92.69 \pm 4.565 \%$.

Table 5. Mean \& standard deviation of variable range Batch Size on 10,000 epochs.

\begin{tabular}{ccccc}
\hline Batch Size & $\mathbf{1 0}$ & $\mathbf{2 0}$ & $\mathbf{5 0}$ & $\mathbf{1 0 0}$ \\
\hline & Mean $(\mu) \pm \mathrm{SD}(\sigma)$ & Mean $(\mu) \pm$ SD $(\sigma)$ & Mean $(\mu) \pm$ SD $(\sigma)$ & Mean $(\mu) \pm$ SD $(\sigma)$ \\
\hline House B & $0.9261 \pm 0.0734$ & $0.9332 \pm 0.0479$ & $0.9327 \pm 0.0413$ & $0.9234 \pm 0.0458$ \\
\hline Kyoto & $0.9407 \pm 0.0620$ & $0.9334 \pm 0.0479$ & $0.9419 \pm 0.0413$ & $0.9394 \pm 0.0458$ \\
\hline
\end{tabular}

Table 6. Mean \& Standard deviation of different Epcohs on 100 Batch Size.

\begin{tabular}{ccccc}
\hline Epochs & $\mathbf{1 0 0 0}$ & $\mathbf{5 0 0 0}$ & $\mathbf{8 0 0 0}$ & $\mathbf{1 0 0 0 0}$ \\
\hline & Mean $(\mu) \pm$ SD $(\sigma)$ & Mean $(\mu) \pm$ SD $(\sigma)$ & Mean $(\mu) \pm$ SD $(\sigma)$ & Mean $(\mu) \pm \operatorname{SD}(\sigma)$ \\
\hline House B & $0.9030 \pm 0.0655$ & $0.930 \pm 0.0468$ & $0.9295 \pm 0.0353$ & $0.9103 \pm 0.0482$ \\
\hline Kyoto & $0.9175 \pm 0.0613$ & $0.9303 \pm 0.0347$ & $0.9388 \pm 0.0304$ & $0.9411 \pm 0.0464$ \\
\hline
\end{tabular}

Table 7. 10-fold Cross-validation Result.

\begin{tabular}{ccc}
\hline & Mean \pm SD Accuracy & Mean \pm SD Error \\
\hline House B & $0.9148 \pm 0.0458$ & $0.476161 \pm 0.15032$ \\
\hline Kyoto & $0.9390 \pm 0.0455$ & $0.31367 \pm 0.21140$ \\
\hline
\end{tabular}

Figure 16 shows the training and testing accuracies of our method. The graph depicts successful training and recognition of activity in accordance. The training loss is comparatively more than test loss, which is natural when the training goes lots of phases to learn the activity's actual occurrence.

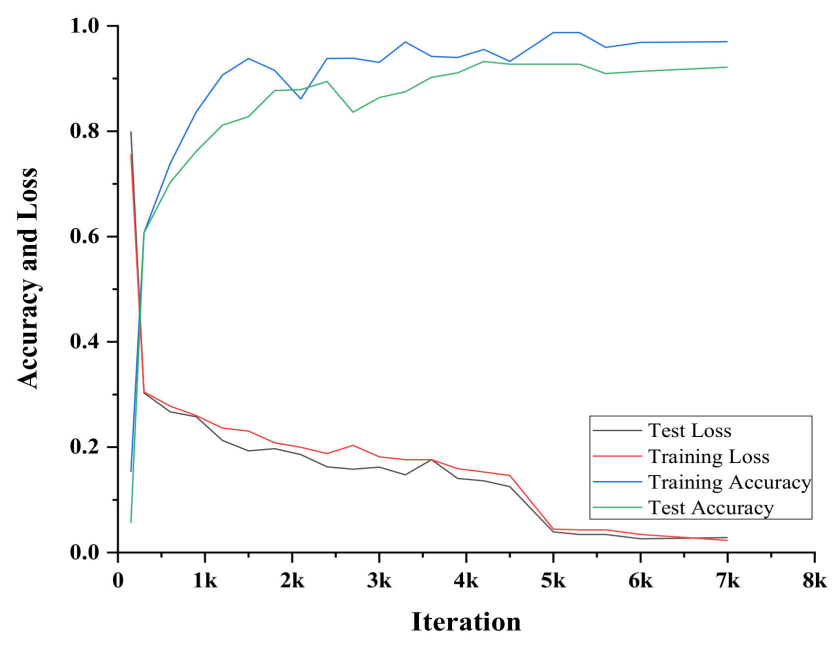

Figure 16. Accuracy of Training or Testing. 
Therefore, the proposed approach recognizes that complex activity, like concurrent and interleaved activity, is better than other techniques. The window size is also an important parameter to consider as too small size may not contain all information and too large causes the vacant producing recognition errors.

Figure 17 shows the accuracy and the F-score of the proposed method compared to the other well-known approaches. Our proposed method outperforms other existing approaches holding accuracy of around $94 \%$ on average. Our proposed method's distinguishing feature is that both the concurrent and interleaved activities are recognized through the single platform; instead, others use a different platform to recognize each activity separately.

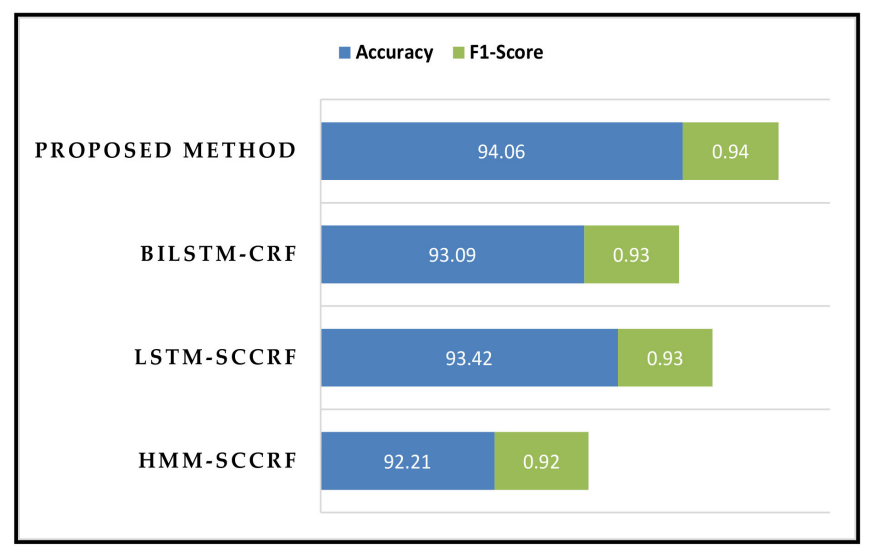

Figure 17. Overall accuracy and F-score of the proposed method.

We did our best to develop an adaptive way to adjust the searching process, reshaping, adding, and removing various layers. However, the excellent approach could be who has perfect generalization. The resident's activities are recognized effectively and wisely by using the binary sensors deployed in smart homes. The external sensors have been used rather than wearable and camera or video sensors to avoid the burden and protect the inhabitant's privacy. In the future, more complex activities like multiple users' activity will be recognition by improving and updating the proposed method. As activity instances and training samples are not much, the technique could be suitable for many data like big data. We will also explore a transfer learning approach to this model to recognize the more complex activity on large-scale data and cloud infrastructures. In conclusion, it would seem preferable to use the model that offers fewer parameter complexities and computational loss.

Author Contributions: Conceptualization, K.T.; methodology, K.T.; software, K.T. and B.L.; validation, K.T. and Z.M.A.A.; formal analysis, K.T. and Z.M.A.A.; investigation, K.T.; resources, Z.M.A.A. and B.L.; data collations, K.T., Z.M.A.A. and B.L.; writing, K.T.; review and editing K.T. and S.-H.Y. ; visualisation, K.T.; supervision, S.-H.Y.; project administration, S.-H.Y.; funding acquisition, S.-H.Y. All authors have read and agreed to the published version of the manuscript.

Funding: This research was conducted by the research grant of AI Home Platform Development Project (2020-0574).

Acknowledgments: This research was supported by the Ministry of Trade, Industry \& Energy (2020-0574), Republic of Korea, and conducted by the Research Grant of Kwangwoon University in 2020.

Conflicts of Interest: The authors declare no conflict of interest.

\section{References}

1. Van Kasteren, T.L.M.; Englebienne, G.; Kröse, B.J.A. An activity monitoring system for elderly care using generative and discriminative models. Pers. Ubiquitous Comput. 2010, 14, 489-498. [CrossRef]

2. Rialle, V.; Duchêne, F.; Noury, N.; Bajolle, L.; Demongeot, J. Health "Smart" Home: Information technology for patients at home. Telemed. e-Health 2002, 8, 395-409. [CrossRef] [PubMed] 
3. Fiorini, L.; Bonaccorsi, M.; Betti, S.; Dario, P.; Cavallo, F. Ambient Assisted Living. Ital. Forum Ambient Assisted Living 2016, 426, 251.

4. Rashidi, P.; Cook, D.J. Keeping the resident in the loop: Adapting the smart home to the user. IEEE Trans. Syst. Man, Cybern. Part A Syst. Humans 2009, 39, 949-959. [CrossRef]

5. Tapia, E.M.; Intille, S.S.; Larson, K. Activity recognition in the home using simple and ubiquitous sensors. Lect. Notes Comput. Sci. 2004, 3001, 158-175.

6. Kim, E.; Helal, S.; Cook, D. Human activity recognition and pattern discovery. IEEE Pervasive Comput. 2010, 9, 48-53. [CrossRef] [PubMed]

7. Hu, D.H.; Yang, Q. CIGAR: Concurrent and interleaving goal and activity recognition. In Proceedings of the Twenty-Third AAAI Conference on Artificial Intelligence, Chicago, IL, USA, 13-17 July 2008.

8. Malcolm, P.; Michael, W.; Viv, J.S.; Rebecca, L.; Sally, M.; David, L.R.; Jeremy, D.; Alison, W. Development and validation of an organizational climate measure. J. Organ. Behav. 2011, 10-26. [CrossRef]

9. Vail, D.L.; Veloso, M.M.; Lafferty, J.D. Conditional random fields for activity recognition. In Proceedings of the 6th International Joint Conference on Autonomous agents and Multiagent Systems-AAMAS'07, Honolulu, HI, USA, 14-18 May 2007.

10. Patterson, D.J.; Liao, L.; Fox, D.; Kautz, H. Inferring high-level behavior from low-level sensors. In Proceedings of the International Conference on Ubiquitous Computing (UbiComp), Seattle, WA, USA, 12-15 October 2003.

11. Kabir, M.H.; Thapa, K.; Yang, J.-Y.; Yang, S.-H. State-space based linear modeling for human activity recognition in smart space. Intell. Autom. Soft Comput. 2018, 1-9. [CrossRef]

12. Hoque, M.R.; Kabir, M.H.; Seo, H.; Yang, S.-H. PARE: Profile-Applied Reasoning Engine for context-aware system. Int. J. Distrib. Sens. Netw. 2016, 12, 1-9. [CrossRef]

13. Sung-Hyun, Y.; Thapa, K.; Kabir, M.H.; Hee-Chan, L. Log-Viterbi algorithm applied on second-order hidden Markov model for human activity recognition. Int. J. Distrib. Sens. Netw. 2018, 14. [CrossRef]

14. Wang, J.; Hu, F.; Li, L. Deep Bi-directional long short-term memory model for short-term traffic flow prediction. Lect. Notes Comput. Sci. 2017, 306-316. [CrossRef]

15. Saon, G.; Kurata, G.; Sercu, T.; Audhkhasi, K.; Thomas, S.; Dimitriadis, D.; Cui, X.; Ramabhadran, B.; Picheny, M.; Lim, L.-L.; et al. English conversational telephone speech recognition by humans and machines. In Proceedings of the Interspeech 2017, 18th Annual Conference of the International Speech Communication Association, Stockholm, Sweden, 20-24 August 2017; pp. 132-136.

16. Vaidya, R.; Trivedi, D.; Satra, S.; Pimpale, M. Handwritten character recognition using deep-learning. In Proceedings of the 2018 Second International Conference on Inventive Communication and Computational Technologies (ICICCT), Coimbatore, India, 20-21 April 2018; pp. 772-775.

17. Arif, S.; Wang, J.; Ul-Hassan, T.; Fei, Z. 3D-CNN-based fused feature maps with lstm applied to action recognition. Futur. Internet 2019, 11, 42. [CrossRef]

18. Sutton, C.; McCallum, A. Piecewise pseudolikelihood for efficient training of conditional random fields. Comput. Sci. Dep. Fac. Publ. Ser. 2007, 62, 863-870.

19. Kautz, R.L. Physics Letters A; Elsevier: Amsterdam, The Netherlands, 1987; Volume 125, pp. $315-319$. ISSN 0375-9601.

20. Maurer, U.; Smailagic, A.; Siewiorek, D.P.; Deisher, M. Activity recognition and monitoring using multiple sensors on different body positions. In Proceedings of the International Workshop on Wearable and Implantable Body Sensor Networks (BSN'06), Cambridge, MA, USA, 3-5 April 2006; Volume 4. [CrossRef]

21. Ponce, H.; Martínez-Villaseñor, L.; Miralles-Pechúan, L. A Novel wearable sensor-based human activity recognition approach using artificial hydrocarbon networks. Sensors 2016, 16, 1033. [CrossRef] [PubMed]

22. Ordóñez, F.J.; Roggen, D. Deep convolutional and LSTM recurrent neural networks for multimodal wearable activity recognition. Sensors 2016, 16, 115. [CrossRef] [PubMed]

23. Yalcin, H.; Yalçın, H. Human activity recognition using deep belief networks. In Proceedings of the 2016 24th Signal Processing and Communication Application Conference (SIU), Zonguldak, Turkey, 16-19 May 2016; pp. 1649-1652.

24. Bevilacqua, A.; Macdonald, K.; Rangarej, A.; Widjaya, V.; Caulfield, B.; Kechadi, T. Human activity recognition with convolutional neural networks. In Machine Learning and Knowledge Discovery in Databases. ECML PKDD, 2018. Lecture Notes in Computer Science; Springer: Cham, Switzerland, 2018; Volume 11053. 
25. Singh, D.; Merdivan, E.; Psychoula, I.; Kropf, J.; Hanke, S.; Geist, M.; Holzinger, A. Human activity recognition using recurrent neural networks. In Machine Learning and Knowledge Extraction. CD-MAKE 2017. Lecture Notes in Computer Science; Holzinger, A., Kieseberg, P., Tjoa, A., Weippl, E., Eds.; Springer: Cham, Switzerland, 2017; Volume 10410.

26. Bao, L.; Intille, S.S. Activity recognition from user-annotated acceleration data most. In Pervasive Computing; Springer: Berlin, Heidelberg, 2004; pp. 1-17.

27. Lee, M.-h.; Kim, J.; Kim, K.; Lee, I.; Jee, S.H.; Yoo, S.K. Physical Activity Recognition Using a Single Tri-Axis Accelerometer; MIT Press: Cambridge, MA, USA, 2009; Volume I, pp. 20-23.

28. Kabir, M.H.; Hoque, M.R.; Thapa, K.; Yang, S.-H. Two-layer hidden markov model for human activity recognition in home environments. Int. J. Distrib. Sensor Netw. 2016, 1-12. [CrossRef]

29. Nazerfard, E.; Das, B.; Holder, L.B.; Cook, D.J. Conditional random fields for activity recognition in smart environments. In Proceedings of the 1st ACM International Health Informatics Symposium, IHI'10, Arlington, VA, USA, 11-12 November 2010; pp. 282-286. [CrossRef]

30. Sutskever, I.; Vinyals, O.; Li, Q. Sequence to sequence learning with neural networks. In Proceedings of the Advances in Neural Information Processing Systems (NIPS 2014), Montreal, QC, Canada, 8-13 December 2014.

31. Graves, A.; Jaitly, N.; Mohamed, A.-R. Hybrid speech recognition with deep bidirectional LSTM. In Proceedings of the Automatic Speech Recognition and Understanding (ASRU), 2013 IEEE Workshop, Olomouc, Czech Republic, 8-12 December 2013; pp. 273-278.

32. Donahue, J.; Hendricks, L.A.; Guadarrama, S.; Rohrbach, M.; Venugopalan, S.; Darrell, T.; Saenko, K. Long-term recurrent convolutional networks for visual recognition and description. In Proceedings of the Computer Vision and Pattern Recognition, Boston, MA, USA, 7-12 June 2015.

33. Karpathy, A.; Joulin, A.; Fei-Fei, L. Deep fragment embeddings for bidirectional image sentence mapping. In Proceedings of the Advances in Neural Information Processing Systems 27: Annual Conference on Neural Information Processing Systems 2014, Montreal, QC, Canada, 8-13 December 2014.

34. Galley, M. A skip-chain conditional random field for ranking meeting utterances by importance. In Proceedings of the Conference on Empirical Methods in Natural Language Processing, Sydney, Australia, 22-23 July 2006; pp. 364-372.

35. Greff, K.; Srivastava, R.K.; Koutnik, J.; Steunebrink, B.R.; Schmidhuber, J. LSTM: A search space odyssey. arXiv 2015, arXiv:1503.04069. [CrossRef] [PubMed]

36. Hammerle, N.Y.; Halloran, S.; Ploetz, T. Deep, convolutional, and recurrent models for human activity recognition using wearables. In Proceedings of the Twenty-Fifth International Joint Conference on Artificial Intelligence, New York, NY, USA, 9-15 July 2016.

37. Sutton, C.; McCallum, A. An introduction to conditional random fields for relational learning. In Introduction to Statistical Relational Learning; Getoor, L., Taskar, B., Eds.; MIT Press: Cambridge, MA, USA, 2006.

38. Van Kasteren, T.L.M. Dataset. Available online: https://sites.google.com/site/tim0306/datasets (accessed on 17 September 2020).

39. CASAS Dataset. Available online: http://casas.wsu.edu/datasets/ (accessed on 17 September 2020).

40. Lee, C. LSTM-CRF Models for Named Entity Recognition. IEICE Trans. Inf. Syst. 2017, D, 882-887. [CrossRef]

41. Huang, Z.; Xu, W.; Yu, K. Bidirectional lstm-crf models for sequence tagging. In Proceedings of the 21st International Conference on Asian Language Processing, Beijing, China, 26-31 July 2015.

(C) 2020 by the authors. Licensee MDPI, Basel, Switzerland. This article is an open access article distributed under the terms and conditions of the Creative Commons Attribution (CC BY) license (http://creativecommons.org/licenses/by/4.0/). 